\title{
Developmental Changes in Electrophysiological Properties of LGNd Neurons during Reorganization of Retinogeniculate Connections
}

\author{
Ary S. Ramoa and David A. McCormick \\ Section of Neurobiology, Yale University School of Medicine, New Haven, Connecticut 06510
}

\begin{abstract}
Changes in electrophysiological properties of neurons in the ferret dorsal LGN (LGNd) were studied during early postnatal life, a critical developmental period when changes occur in morphology, connectivity, and response properties of LGNd neurons. Using the patch-clamp technique to obtain wholecell recordings from cells maintained as in vitro slices of thalamus, several distinctive properties were observed in the immature LGNd. Relatively low resting membrane potentials were present that became more negative during the first 2 postnatal weeks. In addition, immature neurons exhibited high input resistances that decreased during early postnatal development.
\end{abstract}

At all ages postnatally, neurons were capable of generating a train of $\mathrm{Na}^{+}$-dependent action potentials in response to intracellular injection of a depolarizing current pulse. Moreover, immature neurons resembled older cells in that little spike frequency adaptation was present during a train of action potentials. Action potential activity in immature neurons was nevertheless distinctive in several respects: (1) during the first 2-3 postnatal weeks action potentials became shorter in duration and larger in amplitude; (2) during the same period, thresholds for generation of action potentials changed in conjunction with the changes in resting membrane potential, becoming more negative; and (3) plots of frequency versus injected current revealed that thresholds for generation of trains of action potentials were reached with intracellular injection of lower current levels at earlier ages. These findings raise the possibility that relatively weak ionic currents generated at immature synapses have unexpectedly strong effects on the young LGNd neuron.

Finally, immature cells possessed remarkably weak lowthreshold calcium spikes and hyperpolarization-activated currents in comparison to those present in relay cells at the end of the third postnatal week. These results suggest that the intrinsic oscillatory behavior found in adult thalamus, which depends on activation of both types of current, may not be present at early ages.

In conclusion, ferret LGNd neurons display substantial maturation in their electrophysiological properties during the first 2 postnatal weeks, a critical period for reorganization of retinogeniculate connectivity. The electrophysiological

\footnotetext{
Received Apr. 26, 1993; revised Sept. 15, 1993; accepted Sept. 29, 1993.

This work was supported by NIH. We thank Dr. Arnold Kriegstein for his contributions to this project.

Correspondence should be addressed to Ary S. Ramoa, Virginia Commonwealth University, Medical College of Virginia, Department of Anatomy, Box 709, Richmond, VA 23298-0709

Copyright (C) 1994 Society for Neuroscience $0270-6474 / 94 / 142089-09 \$ 05.00 / 0$
}

properties of immature relay cells may contribute to synaptic plasticity mechanisms by enhancing the excitatory responses of LGNd neurons to retinal and cortical input and limiting nonvisual intrinsic and intrathalamic oscillatory activity.

IKey words: lateral geniculate nucleus, ferret, development, patch-clamp technique, electrophysiological properties, slice preparation]

The adult dorsal LGN (LGNd) of ferrets, cats, and primates is characterized by highly specific anatomical connections and electrophysiological properties. Anatomically, the adult LGNd exhibits eye specific laminae (Hickey and Guillery, 1974) that contain neurons belonging to distinct anatomical classes (Guillery, 1966; Sutton and Brunso-Bechtold, 1991). Electrophysiologically, LGNd relay neurons are characterized by two different modes of action potential generation known as burst firing and single spike activity (reviewed in Steriade et al., 1990). The burst firing mode is prevalent during slow wave sleep, a period in which the thalamus is actively involved in the generation of synchronized oscillations, such as spindle waves (reviewed in Steriade et al., 1990) and responsiveness to visual stimulation is poor (Livingstone and Hubcl, 1981). Single spike, or tonic, activity occurs during the waking and attentive state, when visual receptive field organization displays characteristic center and surround mechanisms with high spatial resolving power (Derrington and Fuchs, 1979). Many of these characteristics (e.g., spindle waves and well-defined receptive field properties) are not mature in the newborn animal, but develop prior to and after eye opening (Adrien and Rollwarg, 1974; Daniels et al., 1978: Ikeda and Tremain. 1978; Domich et al., 1987).

Changes in morphology, anatomical connections, and intrinsic membrane properties will all affect the development of LGNd function. Numerous studies have demonstrated that the mature anatomical characteristics of the $\mathrm{LGNd}$ result from extensive modifications in circuit organization (Rakic, 1976; Linden et al., 1981; Shatz, 1983) and, at the single-cell level. growth and remodeling of dendritic trees (Sutton and Brunso-Bechtold, 1991). In contrast, much less is known concerning the development of the electrophysiological properties of the constituent cell types of the LGNd and their synaptic interaction. The intrinsic membrane properties of adult LGNd neurons are remarkably complex in that individual cells display a rich diversity of ionic currents that underlie the different modes of thalamic firing (for a recent review, see McCormick, 1992). In other cell types, such as cortical and hippocampal pyramidal cells. intrinsic membrane properties develop in parallel with cellular morphology and anatomical connections (McCormick and Prince, 1982; Schwartzkroin and Kunkel, 1982). By analogy, these find- 
ings suggest that considerable maturation of electrophysiological properties of LGNd relay cells may also occur postnatally during the period of anatomical rearrangements and therefore contribute to the unique characteristics of LGNd activity in the immature animal. Indeed, anatomical and electrophysiological properties do not develop independently: neuronal form will affect membrane properties and the presence and pattern of neuronal activity appear to be important for the development of neuronal morphology and connections (Shatz and Stryker, 1988; Sretavan et al., 1988).

We have examined the intrinsic membrane properties of developing LGNd cells by recording activity in the in vitro slice preparation using the whole-cell patch-clamp technique (Blanton et al., 1989). We have found that ferret LGNd neurons undergo substantial changes in their intrinsic membrane properties during early postnatal periods. We speculate that such changes may be coordinated to reduce burst firing and facilitate the transmission of information from retina to geniculate at early ages, effects that could contribute to the sculpting of relay cell morphology and connectivity.

\section{Materials and Methods}

Thalamic slices from ferrets ranging in age from the day of birth (postnatal day $0, \mathrm{P} 0$ ) to P54 were prepared as described previously (McCormick and Pape, 1990). The animals were deeply anaesthetized (sodium pentobarbital, $40 \mathrm{mg} / \mathrm{kg}$, i.p.) and killed by decapitation. A block of tissue containing the LGNd was removed and placed in physiological saline at a temperature of $5^{\circ} \mathrm{C}$. Slices were prepared as $400-\mu \mathrm{m}$-thick coronal sections on a Vibratome (Ted Pella, model 1000) and placed in an interface-type recording chamber (Fine Science Tools), where they rested on lens paper. To increase mechanical stability during recording, a piece of nylon mesh was later placed over the slices. The boundaries of the LGNd and the location of the recording, drug application, and electrical stimulation electrodes were made visible by illuminating the slice obliquely through a fiber optic light system and observing the preparation with a low-power dissecting microscope. In some experiments, location and morphology of recorded cells in the LGNd were confirmed by intracellular injection of the fluorescent dye Lucifer yellow (see below). Recordings were obtained in the A or A1 sublaminae. Slices were maintained at $32^{\circ} \mathrm{C}$ and continuously superfused with a solution containing (in mM) $\mathrm{NaCl}(126), \mathrm{KCl}(2.5), \mathrm{MgSO}_{4}$ (1.2), $\mathrm{NaHCO}_{3}(26)$, $\mathrm{NaHPO}_{4}(1.25), \mathrm{CaCl}_{2}(2)$, and dextrose (10), saturated with $95 \% \mathrm{O}_{2}$, $5 \% \mathrm{CO}_{2}$ to a final $\mathrm{pH}$ of 7.4 . Recording started approximately $2 \mathrm{hr}$ after the slices were placed in the recording chamber.

Whole-cell recordings were obtained using the patch-clamp technique as described previously (Blanton et al., 1989). Patch electrodes were pulled from borosilicate glass (World Precision Instruments) on a vertical pipette puller (Narishige) and had tip resistances of 4-8 M 2 . Electrodes were filled with one of the following two solutions: a potassiumbased solution that contained (in mM) potassium gluconate (110), $\mathrm{KCl}$ (10). HEPES buffer (10), sodium EGTA (1), $\mathrm{CaCl}_{2}(0.1), \mathrm{MgCl}_{2}$ (2), NaATP $(2 \mathrm{mM})$, and Na-GTP $(0.2)$, and was maintained at $\mathrm{pH} 7.25$; a cesium-based solution that contained cesium gluconate (120), $\mathrm{NaCl}(10)$, HEPES buffer (10), sodium FGTA (1), $\mathrm{MgCl}_{2}$ (2), $\mathrm{CaCl}_{2}(0.1)$, Na-ATP (2), and Na-GTP $(0.1)$ and was maintained at pH 7.25. The calculated free calcium concentration for these solutions was $0.01 \mu \mathrm{M}$.

To achieve a high-impedance seal, the electrode was brought into close proximity of a neuron, as determined by noting decreases (from $10 \%$ to $50 \%$ of initial value) in the amount of a current required to deliver a small voltage step ( $1 \mathrm{mV})$. Once contact will a cell was made, slight negative pressure was applied to achieve the formation of a gigaohm seal. In this cell-attached configuration we often observed current from spontaneous action potentials or channel openings. Additional suction was applied after a few minutes to rupture the membrane and obtain whole-cell access. Pipette capacitance, series resistance, and wholecell capacitance were carefully monitored and compensated electronically in every cell studied.

Tetrodotoxin (10 $\mu \mathrm{M}$ dissolved in the bathing solution) was applied by delivering pulses of pressure to the rear of a broken micropipette (2-
$5 \mu \mathrm{m}$ tip diameter), the tip of which was placed in the slice near (within $50 \mu \mathrm{m}$ ) the recording site.

Recordings were obtained using an Axopatch-1D amplifier. The head stage carrying the electrode was mounted on a Leitz mechanical micromanipulator. Current and voltage data were digitized using a Neurocorder encoding unit (model DR-484, Neurodata Instruments) and stored on VCR tape for subsequent analysis. The pCLAMP data acquisition program (Axon Instruments) was used to acquire and analyze data off line.

\section{Results}

The electrophysiological properties of ferret LGNd neurons exhibited substantial changes during the first 3 postnatal weeks, the major period of retinogeniculate axon segregation (Linden et al., 1981). Recordings obtained from LGNd neurons at P0 and P30 are shown in Figure 1 and illustrate the basic findings. At $\mathrm{P} 30$, the rich diversity of ionic mechanisms that characterize the adult LGNd neuron was already present. Application of a hyperpolarizing current pulse induced the appearance of a depolarizing "sag" in the response due to activation of the hyperpolarization-activated cation current $I_{h}$ (Fig. 1, P30, sag; McCormick and Pape, 1990). Moreover, a low-threshold spike resulting from activation of the calcium current $I_{\mathrm{T}}$ (Jahnsen and Llinas, 1984a,b; Coulter et al., 1989; Crunelli et al., 1989; Hernandez-Cruz and Pape, 1989), was present at the end of the hyperpolarizing pulse (Fig. 1, P30, $\mathrm{Ca}^{++}$spikes). In contrast, in the neuron of the newborn animal, both sag and low-threshold calcium spikes were either absent or very small in amplitude, indicating that the underlying currents have not yet fully developed at this age (Fig. 1, P0).

The examples in Figure 1 also demonstrate that relay neurons even in newborn animals are capable of generating trains of action potentials in response to a depolarizing current pulse. Action potentials at $\mathrm{PO}$ were, however, lower in amplitude and longer in duration than in the older neuron. Comparison of the recordings reveals that the threshold levels for firing action potentials also changed: the firing threshold in the PO neuron was more depolarized than in the P30 cell. Similarly, the recordings suggest that passive membrane properties may change during development: the resting membrane potential level at $\mathrm{P} 0$ was noticeably more depolarized than at P30.

In the following sections, additional examples and quantitative evidence obtained from studying 99 neurons at ages from P0 to P34 will support the conclusion that properties of LGNd neurons undergo drastic changes during the first 3 postnatal weeks.

\section{Developmental changes in resting membrane properties}

The time course of the changes in resting membrane potential, action potential threshold, input resistance, and membrane time constant is shown in the scatter plots of Figure 2. Geniculate neurons were relatively depolarized at birth, with the resting membrane potential becoming progressively more negative postnatally, reaching adult levels by the third week (Fig. $2 A$ ). During the same time period, the threshold level for firing action potentials also changed substantially (Fig. $2 B$ ), and the difference between the two values (i.e., $\mathrm{mV}$ from rest to threshold) remained relatively unaltered (not shown).

The most substantial changes in input resistance of LGNd neurons occurred during the first 2-3 postnatal weeks, although the input resistances during early postnatal ages were quite variable (Fig. 2C). These changes in input resistance were also as- 
Po
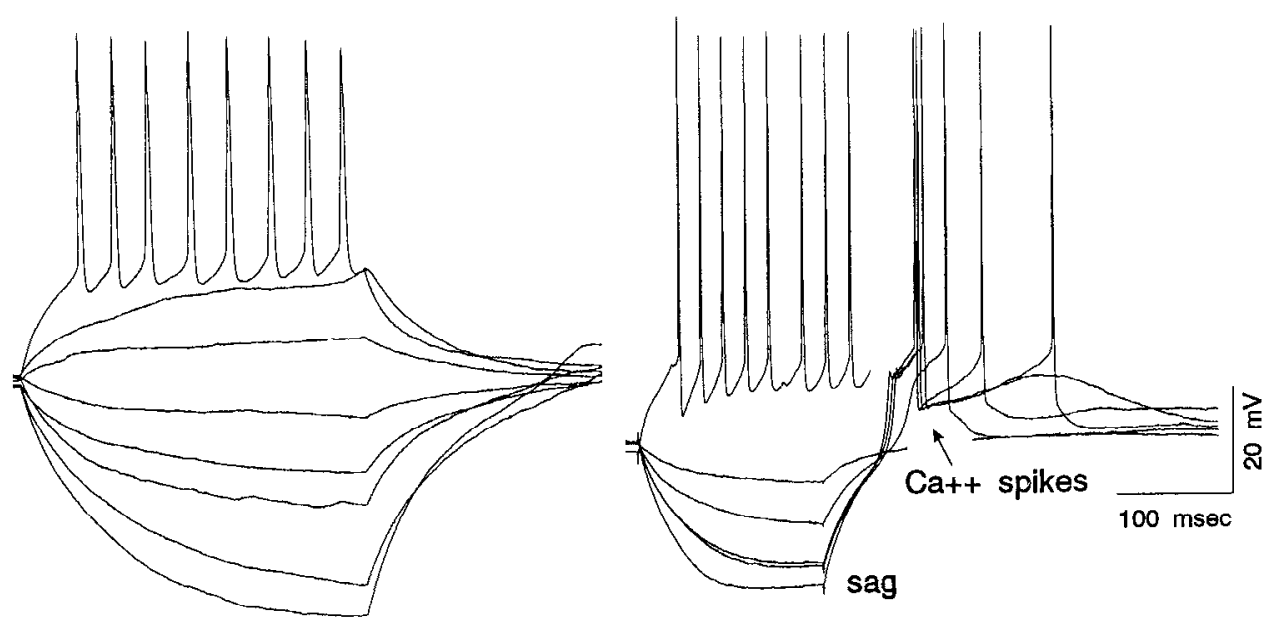

Figure 1. Voltage responses to current pulse injection at $\mathrm{PO}$ and $\mathrm{P} 30$. Responses to the same amplitude of intracellular current injection at $\mathrm{PO}$ displayed longer time constants and larger voltage deflections than at $\mathrm{P} 30$, thus indicating the presence of higher input resistance in the younger neuron. Additional differences between the two cells included a depolarizing sag in the response to a hyperpolarizing pulse at P30 and a low-threshold calcium spike after release from hyperpolarization at $\mathrm{P} 30$ but not at PO. Note also the more depolarized level of the resting membrane potential at $\mathrm{P} 0$ (approximately $-45 \mathrm{mV}$ ) relative to $P 30(-63 \mathrm{mV})$. sociated with a slight trend toward decreased time constants, during the first postnatal month $(r=-0.37 ; p<0.05)$.

\section{Developmental changes in action potential activity}

Single action potentials. Examples of single action potentials recorded at P0 and P23 are shown in Figure 3 to illustrate that action potentials in young neurons were different from those of mature cells in several respects. Action potentials at $\mathrm{P} 0$ were smaller in peak amplitude and longer in duration, and had slower rates of rise and fall than those observed in the more mature neuron. To estimate the time course of these changes, duration and amplitude were computed for the whole population of cells and plotted as a function of age (Fig. 3). The plots show that spikes progressively decreased in duration and increased in amplitude. As in the case of the other parameters examined in this report, changes occurred mainly during the first 2 or 3 postnatal weeks.

In view of the age-related modifications in kinetic properties of action potentials, we have investigated the ionic basis of action potential generation in immature neurons. Intracellular injections of depolarizing current pulses were applied before and after local application of the selective sodium channel blocker TTX $(10 \mu \mathrm{M})$. A typical recording obtained from a P4 neuron using potassium-based pipette solution is illustrated in Figure $4 A$. Application of TTX completely blocked action potential activity observed with the potassium pipette solution, indicating that fast spikes are critically dependent on the activation of sodium channels even at early postnatal ages.

Recordings obtained with micropipettes containing cesium to block potassium currents revealed depolarization-activated slow potentials that probably involve calcium conductances (Jahnsen and Llinas, 1984a,b; McCormick and Prince, 1987). As shown in Figure $4 B$, injection of a depolarizing current pulse evoked a spike lasting scveral hundred milliscconds. Application of TTX only affected the initial, fast depolarizing phase of the slow action potential. These presumed high-threshold calcium spikes
A

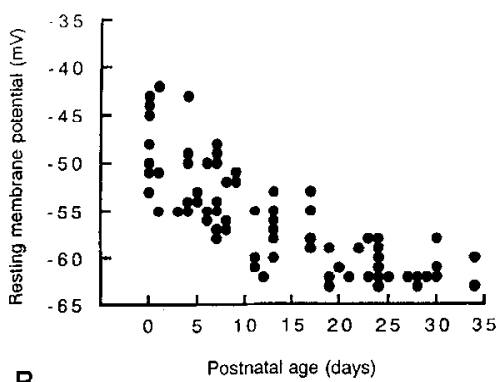

B

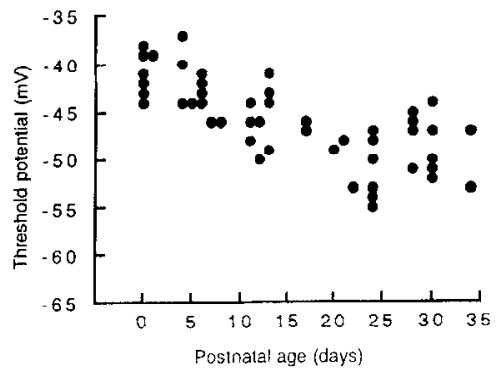

C
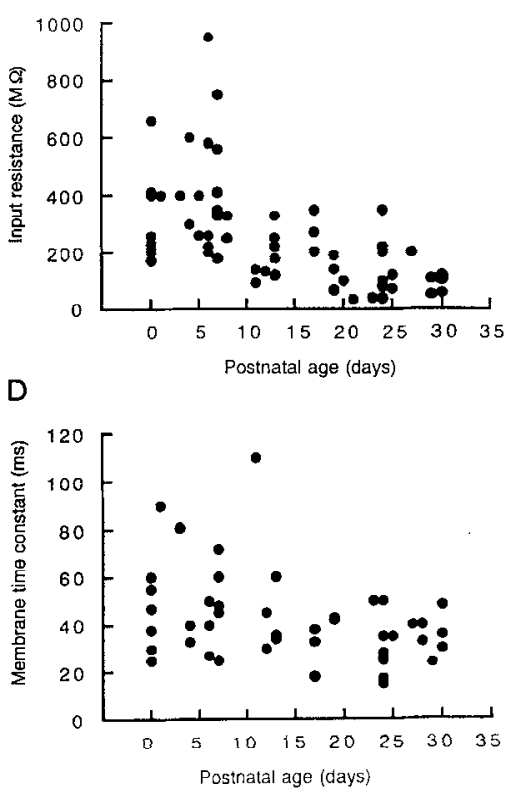

Figure 2. Scatter plots showing agerelated changes in the values of resting membrane potential $(A)$, threshold potential for firing of a single action potential $(B)$, input resistance $(C)$, and membrane time constant $(D)$. Values of resting membrane potentials and threshold potential became progressively more negative during the first 23 postnatal weeks until the adult state was achieved. The similar time course of changes in these parameters indicates that the average deflection in potential required to activate spikes remained relatively stable during postnatal development. There is also a clear trend toward relatively lower values of input resistance with age. Cells recorded using the solution containing potassium gluconate. 


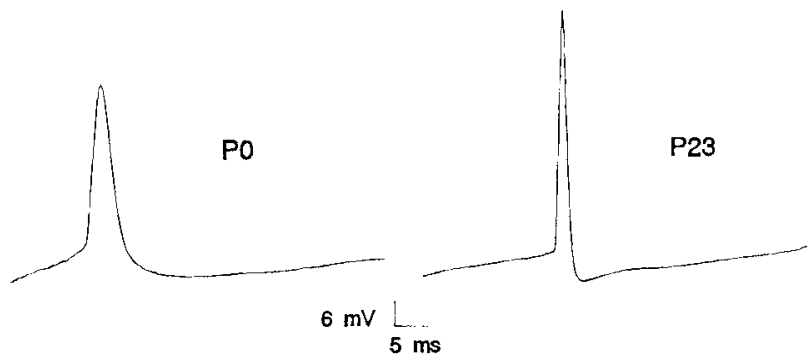

Figure 3. Examples of intracellularly recorded action potentials in immature $(P 0)$ and mature $(P 23)$ animals. Scatter plots below these recordings show the values of amplitude and duration (measured at half-height) for action potentials recorded from each cell in the study. Note the clear increase in amplitude and concomitant decrease in duration of action potentials with age. The first 3 postnatal weeks encompass a stage of development during which changes occur rapidly.
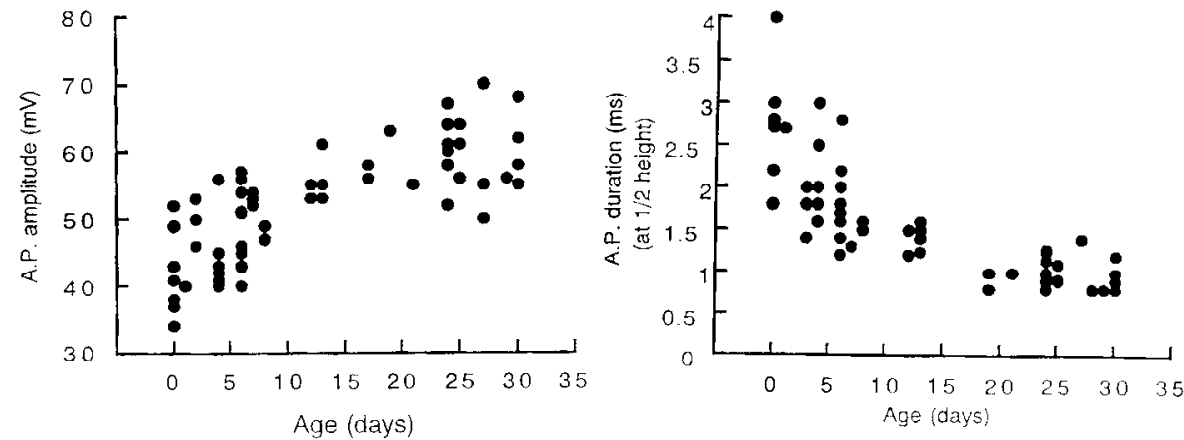

were not a transient feature of LGNd development, since they could also be elicited in older neurons when potassium conductances were blocked (not shown). These results indicate that action potentials at all postnatal ages are mediated largely by voltage-dependent sodium channels with a possible contribution of high-threshold calcium currents.

Trains of action potentials. Intracellular injection of depolarizing current pulses into LGNd neurons could evoke trains of fast action potentials at all ages studied (P0-P35). Examples of repetitive firing at $\mathrm{P} 5$ and $\mathrm{P} 23$ in response to different depolarizing current pulses are shown in Figure $5 \mathrm{~A}$. Increasing the amplitude of the depolarizing current reduced the latency of the first spike and increased firing frequency. Trains of action po- tentials in both neurons exhibited little spike frequency adaptation; that is, the frequency of spikes remained relatively unaltered during the whole duration of a given spike train.

This conclusion is confirmed by examination of the plots of frequency versus interspike interval number (Fig. 5C). The P5 and P23 cells are remarkably similar in showing little spike frequency adaptation. In addition, plots of frequency at the first interspike interval as a function of injected current ( $f-I$ plots) arc also shown in Figure $5 B$ for immature (P0-P6) and older (P23-P28) neurons. The input-output relationships in younger neurons overlapped with those present in more mature neurons, the distinguishing feature being a lower-threshold current level for spike activation at early ages. Quantitative evidence for this
Figure 4. Ionic mechanisms of action potential generation in P4 LGNd neurons. $A$, Recordings using a micropipette solution containing potassium gluconate. Intracellular injection of a depolarizing current pulse resulted in a typical train of action potentials. Local application of tetrodotoxin $(T T X ; 10$ $\mu \mathrm{M}$ inside the pipette solution) completely blocked the action potentials. $B$, Recordings with a solution containing cesium gluconate to block potassium currents. Injection of a depolarizing current pulse resulted in the generation of a slow action potential lasting several hundreds of milliseconds. Local application of TTX reduced the fast rising phase of the spike, while leaving the slower component unaffected, therefore suggesting a significant contribution by a high-threshold calcium current. $\begin{array}{lll}\text { Control } & \text { P4 } 4 X\end{array}$
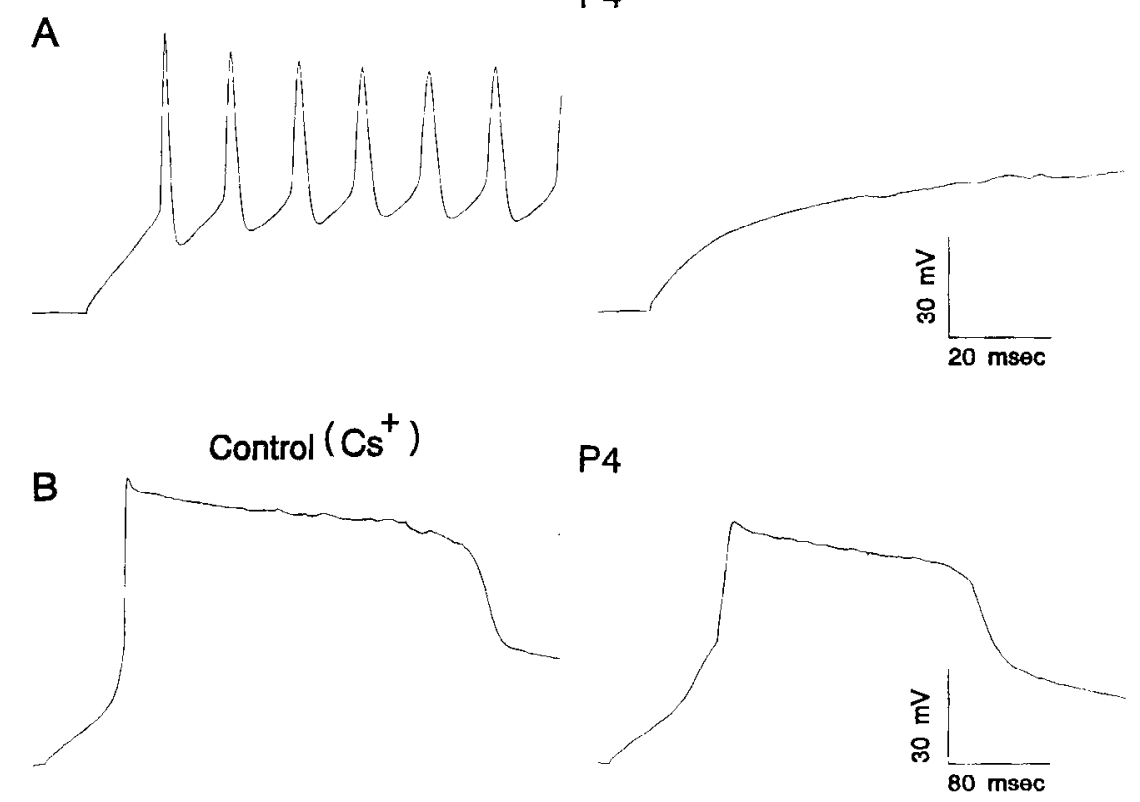

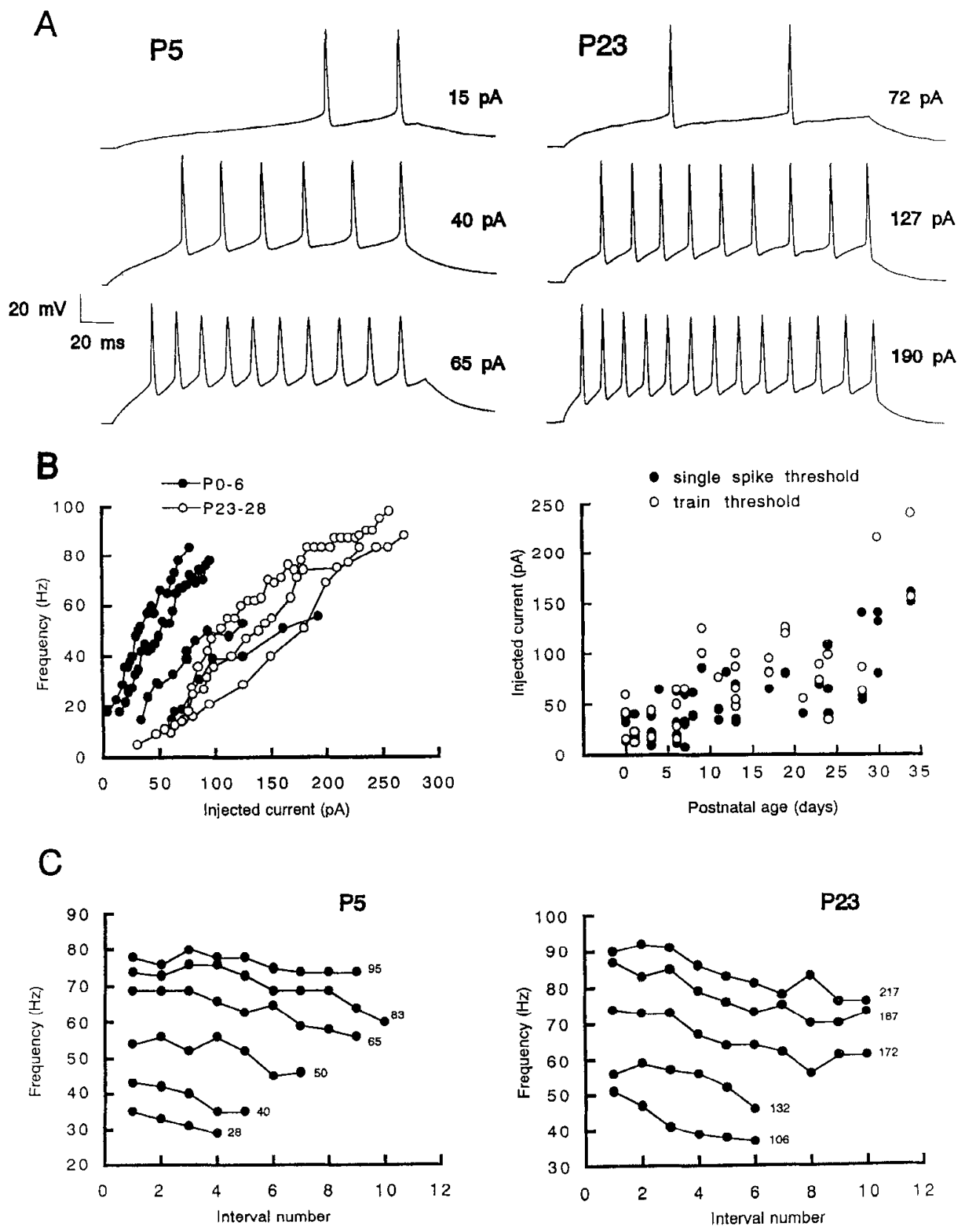

Figure 5. Characteristics of action potential activity in LGNd cells studied at different ages. $A$, Examples are shown of responses of LGNd cells at $\mathrm{P} 5$ and P23 to depolarizing current injections of increasing amplitudc. Note the different range of amplitudes required to generate trains of action potentials at P5 (15-65 pA) and P23 (72-190 pA). $B$, Frequency versus injected current plots for the first interspike interval are shown to the left. Recordings were obtained from animals during the first postnatal week (solid sumbols) and fourth postnatal week (open symbols). To the right is shown the amplitude of injected current needed to generate a single spike (solid symbols) or a train of at least two spikes (open symbols) in each cell. Note the increase in current amplitude with age. $C$, Firing frequency versus interval number for a P5 and a P23 neuron. At the intensities of current injected (indicated to the right of each trace), the neurons exhibited little spike frequency adaptation.

suggestion is provided in the plot of injected current levels required to elicit single spikes (solid symbols) or trains of at least two spikes (open symbols) as a function of age (Fig. 5B).

\section{Developmental changes in low-threshold calcium spikes}

Low-threshold calcium spikes, known to be prominent in adult LGNd relay neurons (Jahnsen and Llinas, 1984a,b), were recorded at resting membrane potential by application of hyperpolarizing current pulses. Figure $6 \mathrm{~A}$ illustrates examples of lowthreshold calcium spikes recorded at $\mathrm{P} 0, \mathrm{P} 7$, and $\mathrm{P} 19$ using the potassium-based pipette solution. Early in development (P0), low-threshold calcium spikes were weak and did not depolarize the membrane sufficiently to generate a burst of action potentials. At P7, these calcium spikes were larger in amplitude, generating enough depolarization to elicit single action potentials, and later, at P19, bursts of two to six action potentials.
We have further estimated the effects of age-dependent increase in calcium spike amplitude by counting the number of action potentials evoked by the calcium spikes in each cell and the results are pooled together in the histogram of Figure $6 \mathrm{~A}$. To standardize the findings, we compared results obtained at resting membrane potential levels after the application of current pulses that hyperpolarized the cell to approximately -90 $\mathrm{mV}$, where removal of inactivation of $I_{\mathrm{T}}$ is relatively complete (Coulter et al., 1989). During the first postnatal week approximately half of the cells studied did not generate calcium spikes or generated such weak calcium currents that action potentials were not elicited. Most of the remaining immature cells during this time generated a single action potential during the small rebound calcium spike. In contrast, during the fourth postnatal week each low-threshold calcium spike generated enough current to activate at least one and up to six action potentials.

To examine calcium spikes in the absence of contaminating 


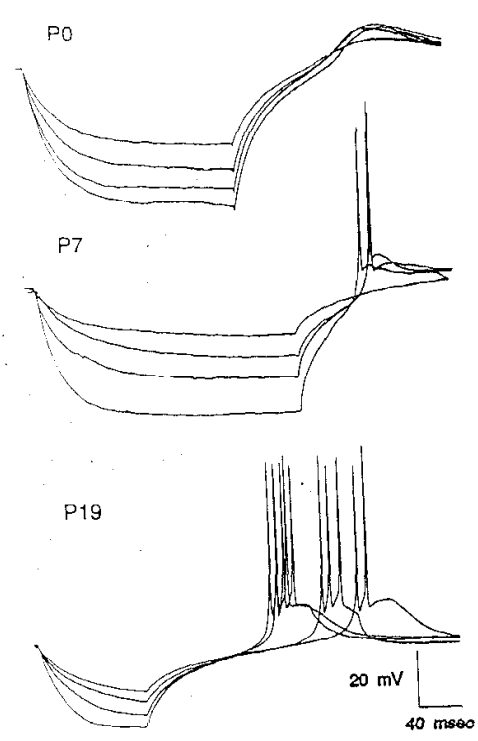

\section{A Potassium solution}

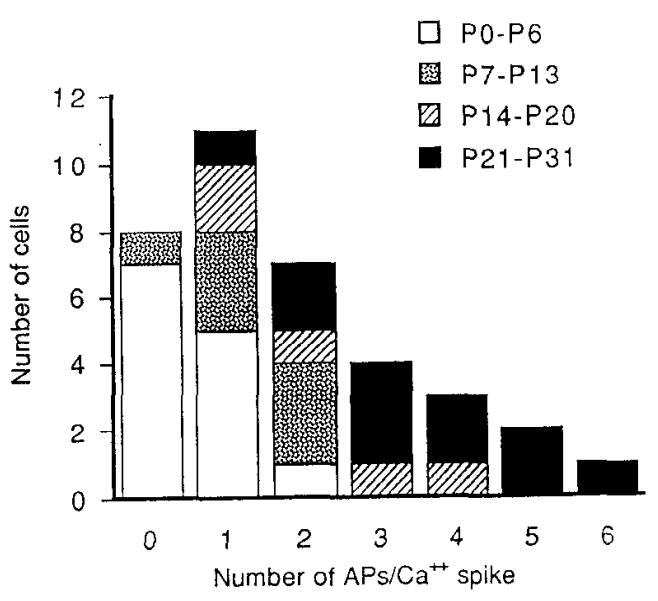

B Cesium solution (TTX)

PO
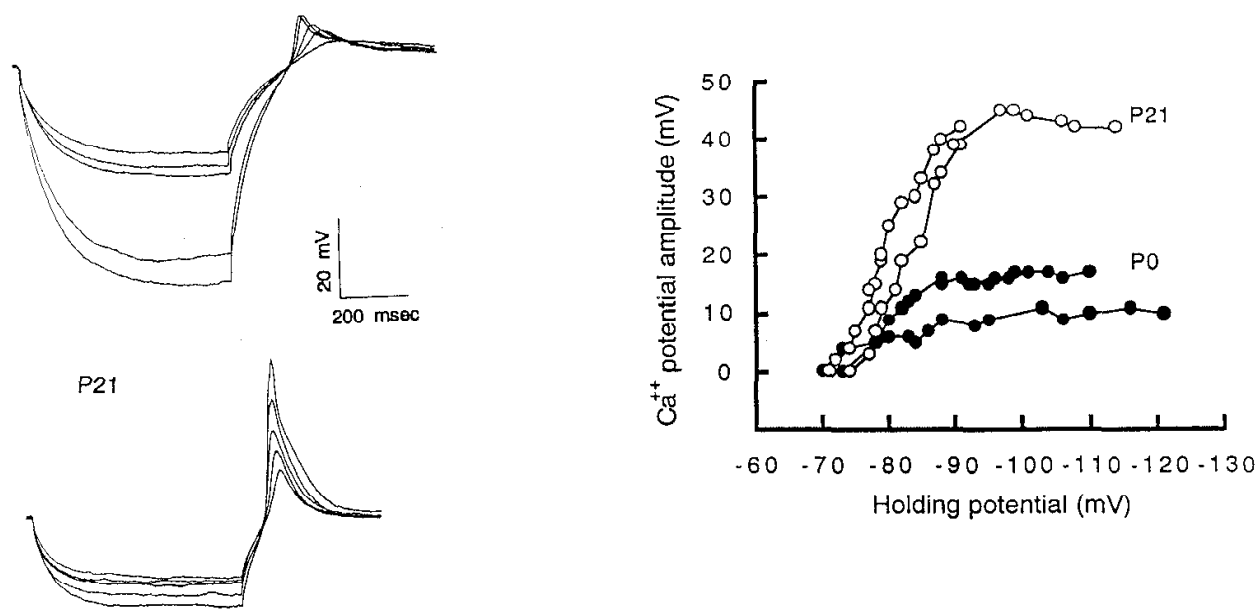

Holding potential $(\mathrm{mV})$

Figure 6. A, Bursting in thalamic neurons studied at different ages using the potassium-based recording solution. Release from hyperpolarization was followed by low-threshold calcium spikes in cells studied at P0, P7, and P19. Note, however, the substantial increase in the amplitude of the calcium spike with age. As a result the number of action potentials generated by each calcium-dependent depolarization also increased with age. Stimulation of the immature neurons with longer-lasting current pulses than at P19 was not sufficient to reveal a strong rebound calcium spike. Recordings were conducted at the resting membrane potential for each cell (P0, $-51 \mathrm{mV} ; \mathrm{P} 7,-55 \mathrm{mV} ; \mathrm{P} 19,-62 \mathrm{mV})$. The histogram pools results from all cells studied with hyperpolarizing pulses to demonstrate that the number of action potentials activated by each calcium spike increased with age. Results included in this histogram were obtained from cells studied with hyperpolarizing current pulses to a holding potential level of approximately $-90 \mathrm{mV} . B$, Examples of low-threshold calcium spikes in neurons recorded with cesium-based pipette solution to block potassium currents, and in the presence of TTX to block fast $\mathrm{Na}^{+}$-dependent action potential activity. The amplitude of calcium spikes elicited in response to increasing amplitude hyperpolarizing pulses (duration $600 \mathrm{msec}$ ) is markedly increased at P21 in comparison with P0 (upper traces). Plot of calcium spike amplitude versus membrane potential achieved during the hyperpolarization (holding potential) from two different cells at each age is also illustrated.

sodium and potassium currents, recordings were conducted using the cesium-based pipette solution and in the presence of 'ГTX. Blocking sodium and potassium conductances enabled a more direct comparison of the low-threshold calcium spikes at different ages. Examples of the results observed at P0 and P21 are shown in Figure $6 B$. At both ages the amplitude of the lowthreshold calcium spike and slope of the depolarizing phase were related to the strength of the preceding hyperpolarizing current pulse. However, the maximum amplitude observed at P2 I was much greater than at P0. Plots of calcium spike amplitude as a function of the membrane potential obtained with a hyperpolarizing pulse (Fig. 6B) also revealed that there is a significant increase in the maximum amplitude of low-threshold calcium spikes with age; the maximum depolarization induced by the calcium currents was three to four times larger at P21 than at $\mathrm{P} 0$. 


\section{Discussion}

The present report demonstrates that the first 3 postnatal weeks in the ferret are charactcrized by several remarkable changes in electrophysiological properties of LGNd relay neurons: (1) lowthreshold $\mathrm{Ca}^{2+}$ spikes become progressively larger and in neurons from newborn animals are typically unable to generate burst discharges; (2) the resting membrane potential becomes progressively more negative, reaching the adult level by the end of the third week; (3) input resistance decreases substantially; (4) action potentials decrease in duration and increase in annplitude; (5) the threshold potential for generation of action potentials becomes progressively more negative; and (6) significantly, trains of action potentials in young neurons are elicited by application of lower levels of current than required in mature cells.

Concurrently with these changes in intrinsic electrophysiological properties of developing LGNd relay neurons, neuronal form, as well as intra- and extrageniculate synaptic connections, is being determined. During the first few postnatal weeks, dendritic dimensions and complexity increase markedly and by $\mathrm{P} 21$ neurons can already be recognized as belonging to one of the adult classes (Sutton and Brunso-Bechtold, 1991). This is also the major period of maturation of retinogeniculate connectivity in the ferret (Linden et al., 1981). These various features of development of the LGNd are not independent. Recent studies suggest that anatomical, morphological, and electrophysiological features of the developing visual system are interrelated, a topic we will discuss below.

Comparison with other studies. In a recent in vitro study of the development of membrane properties of ferret LGNd cells, relay cells after the age of approximately P10 were found to be relatively similar to those of the adult (White and Sur, 1992). Our results also suggest that after the second postnatal week, the basic electrophysiological features of LGNd relay cells are present, although we extended this finding by demonstrating that substantial changes occur in the first 2 postnatal weeks, during the period of segregation of retinal afferents according to eye of origin (Linden et al., 1981).

Although LGNd relay cells were capable of generating sustained trains of action potentials upon depolarization at all ages, they were largely incapable of generating rebound low-threshold $\mathrm{Ca}^{2+}$ spikes during the first postnatal week, suggesting that intrathalamic rhythms may be small or absent in the newborn ferret (e.g., see Adrien and Roffwarg, 1974; Domich et al., 1987; Ramoa et al., 1993). Indeed, another study of the electrophysiological properties of LGNd cells in developing mammals yielded results consistent with our findings: the low-threshold calcium current in thalamocortical cells was found to increase in amplitude during postnatal periods in the cat (Pirchio et al., 1990). The changes were smaller, however, than those of the present study. The differences may be explained by the fact that cats are born considerably more mature than ferrets (by approximately 3 weeks), having completed segregation of the retinogeniculate connections according to eye of origin well before birth (Shatz, 1983).

Investigations of the development of electrophysiological properties in neurons of several other CNS structures have revealed results that are consistent with our present findings in the LGNd. For instance, lower membrane potentials of immature central neurons have been previously reported (Schwartzkroin and Kunkel, 1982; McCormick and Prince,
1987). Moreover, action potentials have been shown to become bricfer in duration and larger in amplitude while the input resistance decreases during postnatal development of rat neocortex (McCormick and Prince, 1987), hippocampus (Schwartzkroin and Kunkel, 1982), cerebellum (Gardette et al., 1985), and spinal cord (Westbrook and Brenneman, 1984; Walton and Fulton, 1986; Fulton, 1987). The characteristic age-dependent modifications in action potentials in the neocortex result mainly from changes in sodium current density related to an increase in the number of channels per unit membrane area (Huguenard et al., 1988), although changes in kinetic properties of the ion channels involved, as well as in their types, may also play a role as they do in other structures (Skaliora et al., 1993). Developmental changes in the voltage dependence of sodium conductances have also been previously described in fetal rat diencephalic neurons (Park and Ahmed, 1991) and could underlie our observations that voltage dependence of action potential generation in the LGNd changes with age.

The development of calcium currents has been studied in several systems (Spitzer, 1991), but specially important in the case of LGNd neurons is the development of the low-threshold calcium current, $I_{\mathrm{T}}$, that underlies the characteristic burst firing behavior of thalamocortical relay cells (Deschenes et al., 1984; Jahnsen and Llinas, 1984a,b). The peculiar late maturation of low-threshold calcium currents in the LGNd may be relevant in retinogeniculate plasticity by reducing burst firing of thalamic cells during early development. It is interesting to note that in other neuronal types, in contrast to LGNd neurons, this calcium current has been found to decrease during development (Kostyuk et al., 1986; McCobb et al., 1989).

These studies of electrophysiological properties during development of different neural structures provide examples of changes that resemble in some respects those found in the LGNd. However, taken together the reports also indicate that immature neural sites display distinctive combinations of intrinsic membrane properties.

Relevance to intrinsic oscillatory behavior in the developing $L G N d$. Mature thalamic relay neurons are known to generate action potentials in two different modes: rhythmic burst firing, in which the generation of a low-threshold calcium spike leads to bursts of two to eight action potentials, and tonic firing, in which trains of single action potentials are elicited (reviewed in Stcriade ct al., 1990). Rhythmic burst firing in adult thalamus occurs during three distinct states: (1) as an expression of intrinsic membrane properties of individual relay neurons (McCormick and Pape, 1990; Steriade et al., 1990), (2) as periodic rebound activity resulting from barrages of IPSPs arriving from the nucleus reticularis of the thalamus during the generation of spindle waves (reviewed in Steriade et al., 1990), and (3) as strong burst activity during the generation of absence seizures (reviewed in Avoli et al., 1990). The reduced levels of lowthreshold calcium current observed in immature neurons in vitro suggest that the burst firing mode typical of the adult thalamus is not present during the first few postnatal weeks in vivo, and predict that all three of these different modes of rhythmic burst firing will be absent during early postnatal development. Indeed, a previous in vivo study of the postnatal activity of cat LGNd relay cells suggested a significant lack of burst activity (which is mediated by the low-threshold $\mathrm{Ca}^{2}$ current) in slow wave sleep during the first few postnatal weeks (Adrien and Roffward, 1974). Preliminary results from our laboratory confirm our prediction: slow intrinsic oscillations and spindle waves typical of 
the mature LGNd (von Krosigk et al., 1993) appear relatively late during postnatal development of the ferret (Ramoa et al., 1993). Investigations of absence seizures in humans, which are thought to be critically dependent upon rhythmic burst firing in the thalamus for their expression, demonstrate that this type of epilepsy is not seen in newborn humans and rarely develops prior to 3-5 years of age (reviewed in Avoli et al., 1990). The protracted development of this abnormal pattern of thalamocortical activity suggests the presence of additional alterations in normal function other than those of development of the lowthreshold $\mathrm{Ca}^{2+}$ spike described here. Additional investigations will be required to determine whether other types of rhythmic oscillatory behavior may be present in the immature LGNd in vivo, since oscillatory behavior found in the immature retina may conceivably lead to synchronized activity of neurons in target nuclei (Meister et al., 1991).

Relevance of changes in membrane properties to excitatory neurotransmission and plasticity during development. The activity of neurons in the developing CNS could contribute to remodeling of connectivity by selective strengthening and weakening of synapses (Hebb, 1949). Some predictions may be proposed to illustrate how the age-related changes in electrophysiological properties of LGNd neurons reported here may enhance Hebbian-type synaptic mechanisms during the critical period of retinogeniculate rearrangements. For instance, both the increased input resistance and lack of intrinsic oscillatory behavior (see above), in combination with the lower current level needed to reach firing threshold in immature cells, raise the possibility that excitatory transmission is enhanced in the immature retinogeniculate and corticogeniculate systems. Another important factor may be the depolarized levels of the resting membrane potential at early postnatal ages, which may contribute to an increased duration of excitatory postsynaptic potentials at the immature retinogeniculate synapse (Ramoa and McCormick, 1994). Enhanced excitatory transmission may be especially important at a time when synapses probably release fewer quanta of neurotransmitter per action potential than in the adult (Campbell and Shatz, 1992).

It is also probable that the more prolonged action potential found in immature LGNd neurons will allow greater entry of cations, including calcium, than would occur if the action potentials were short in duration, as in mature cells (see Spitzer, 1991). Calcium ions can also enter cells through high- and lowthreshold calcium-based action potentials, such as those shown in Figures 4 and 6 in the present report (see also Franz et al., 1986). Although LGNd neurons are not known to fire highthreshold calcium spikes under normal conditions, it is likely that $\mathrm{Ca}^{2+}$ influx during membrane depolarization may play a role in the control of several aspects of nerve activity (for a review, see Miller, 1987). In view of the developmental roles proposed for calcium ions (Lipton and Kater, 1989), it will be important to study further how entrance of calcium into the immature LGNd neurons is regulated.

\section{References}

Adrien J, Roffwarg HP (1974) The development of unit activity in the lateral geniculate nucleus of the kitten. Exp Neurol 43:261-275.

Avoli M, Gloor P, Kostopoulos G, Naquet R (1990) Generalized epilepsy: neurobiological approaches. Boston: Birkhauser.

Blanton MG, LoTurco JJ, Kriegstein AR (1989) Whole cell recording from neurons in slices of reptilian and mammalian cerebral cortex. J Neurosci Methods 30:203-210.

Campbell G, Shatz CJ (1992) Synapses formed by identified retino- geniculate axons during the segregation of eye input. J Neurosci 12 : $1847-1858$.

Coulter DA, Huguenard JR, Prince DA (1989) Calcium currents in rat thalamocortical relay neurons: kinetic properties of the transient, low threshold current. J Physiol (Lond) 414:587-604.

Crunelli V, Lightowler S, Pollard CE (1989) A T-type Ca ${ }^{2+}$ current underlies low-threshold $\mathrm{Ca}^{2+}$ potentials in cells of the cat and rat lateral geniculate nucleus. J Physiol (Lond) 413:543-561.

Daniels JD, Pettigrew JD, Norman JL (1978) Development of singleneuron responses in kitten's lateral geniculate nucleus. J Neurophysiol 41:1373-1393.

Derrington AM, Fuchs AF (1979) Spatial and temporal properties of $\mathrm{X}$ and $\mathrm{Y}$ cells in the cat lateral geniculate nucleus. $\mathrm{J}$ Physiol (Lond) 293:347-364.

Deschenes M, Paradis M, Roy JP, Steriade M (1984) Electrophysiolugy of neurons of lateral thalanic nuclei in cal: resting membrane properties and burst discharges. J Neurophysiol 51:1196-1219.

Domich L, Oakson G, Deschenes M, Steriade M (1987) Thalamic and cortical spindles during early ontogenesis in kittens. Dev Brain Res 31:140-142.

Franz P, Galvan M, Constanti A (1986) Calcium-dependent potentials and associated inward currents in guinea-pig neocortical neurons in vitro. Brain Res 366:262-271.

Fulton BP (1987) Postnatal changes in conduction velocity and soma action potential parameters of rat dorsal root ganglion neurones. Neurosci Lett 73:125-130

Gardette R, Debono M, Dupont JL, Crepel F (1985) Electrophysiological studies on the postnatal development of intracerebellar nuclei neurons in rat cerebellar slices maintained in vitro. II. Membrane conductances. Dev Brain Res 20:97-106.

Guillery RW (1966) A study of Golgi preparations from the dorsal lateral geniculate nucleus of the adult cat. J Comp Neurol 128:2150.

Hebb DO (1949) The organization of behavior. New York: Wiley.

Hernandez-Crus A, Pape HC (1989) Identification of two calcium currents in acutely dissociated neurons from the rat lateral geniculate nucleus. J Neurophysiol 61:1270-1283.

Hickey TL, Guillery RW (1974) An autoradiographic study of retinogeniculate pathways in the cat and fox. J Comp Neurol 156:239254.

Huguenard JR, Hamill OP, Prince DA (1988) Developmental changes in $\mathrm{Na}^{+}$conductances in rat neocortical neurons: appearance of a slowly inactivating component. J Neurophysiol 59:778-795.

Ikeda $\mathrm{H}$, Tremain KE (1978) The development of spatial resolving power of lateral geniculate neurones in kittens. Exp Brain Res 341: 193-206.

Jahnsen H, Linas R (1984a) Electrophysiological properties of guineapig thalamic neurons: an in vitro study. J Physiol (Lond) 349:205226.

Jahnsen H, Llinas R (1984b) Ionic basis for the electroresponsiveness and oscillatory properties of guinea-pig thalamic neurons in vitro. $\mathrm{J}$ Physiol (Lond) 349:227-247.

Kostyuk PG, Fedulova SA, Veselovskii NS (1986) Changes in the ionic mechanisms of the electrical excitability of rat sensory neuronal membrane during ontogenesis. Distribution of ionic channels carrying inward currents. Neurophysiology 18:813-820.

Linden DC, Guillery RW, Cucchiaro J (1981) The dorsal lateral geniculate nucleus of the normal ferret and its postnatal development. J Comp Neurol 203:189--211.

Lipton SA, Kater SB (1989) Neurotransmitter regulation of neuronal outgrowth, plasticity and survival. Trends Neurosci 12:265-269.

Livingstone MS, Hubel DH (1981) Effects of sleep and arousal on the processing of visual information in the cat. Nature 291:554-561.

McCobb DP, Best PM, Beam KG (1989) Development alters the expression of calcium currents in chick limb motoneurons. Neuron 2:1633-1643

McCormick DA (1992) Neurotransmitter actions in the thalamus and cerebral cortex and their role in neuromodulation of thalamocortical activity. Prog Neurobiol 39:337-388

McCormick DA, Pape HC (1990) Properties of a hyperpolarizationactivated cation current and its role in rhythmic oscillation in thalamic relay neurones. J Physiol (Lond) 431:291-318.

McCormick DA, Prince DA (1987) Post-natal development of electrophysiological properties of rat cerebral cortical pyramidal neurones. J Physiol (Lond) 393:743-762. 
Meister M, Wong ROL. Baylor DA, Shatz CI (1991) Synchronous bursts of action potentials in ganglion cells of the developing mammalian retina. Science 252:939-943.

Miller R (1987) Multiple calcium channels and neuronal function. Science 235:46-52.

Park CC, Ahmed Z (1991) Characterization of sodium currents in developing rat diencephalic neurons in serum-free culture. J Neurophysiol 65:1011-1021.

Pirchio M, Lightowler S, Crunelli V (1990) Postnatal development of the $T$ calcium current in cat thalamocortical cells. Neuroscience 38 : $39-45$.

Rakic P (1976) Prenatal genesis of connections subserving ocular dominance in the rhesus monkey. Nature 261:467-471.

Ramoa AS, McCormick DA (1994) Enhanced activation of NMDA receptor responses at the immature retinogeniculate synapse. J Neurosci 14:2098-2105.

Ramoa AS, Trent F, McCormick DA (1993) Synchronized neuronal firing in the developing ferret LGNd. Soc Neurosci Abstr 19:240.

Schwartzkroin PA, Kunkel DD (1982) Electrophysiology and morphology of the developing hippocampus of fetal rabbits. J Neurosci $2: 448-462$

Shatz CJ (1983) The prenatal development of the cat's retinogeniculate pathway. J Neurosci 3:482-499.

Shatz CJ, Stryker MP (1988) Prenatal tetrodotoxin infusion blocks segregation of retinogeniculate afferents. Science 242:87-89.

Skaliora 1, Scobey RP. Chalupa LM (1993) Prenatal development of excitability in cat retinal ganglion cells: action potentials and sodium currents. J Neurosei 13:313-323.

Spitzer NC (1991) A developmental handshake: neuronal control of ionic currents and their control of neuronal differentiation. J Neurobiol 22:659-673.

Sretavan DW, Shatz CJ, Stryker MP (1988) Modification of retinal ganglion cell axon morphology by prenatal infusion of tetrodotoxin. Nature 336:468-471

Steriade M, Jones EG, Llinas RR (1990) Thalamic oscillations and signalling. New York: Wiley.

Sutton JK, Brunso-Bechtold JK (1991) A Golgi study of dendritic development in the dorsal lateral geniculate nucleus of normal ferrets. J Comp Neurol 309:71-85.

von Krosigk M, Bal T, McCormick DA (1993) Cellular mechanisms of a synchronized oscillation in the thalamus. Science 261:361-364.

Walton K. Fulton BP (1986) The ionic mechanisms underlying the firing properties of rat neonatal motoneurones studied in vitro. Neuroscience 19:669-683.

Westbrook GL, Brenneman DE (1984) The development of spontaneous activity in spinal cord culture. In: Developmental neuroscience: pharmacological and clinical aspects (Caciagli F, Giacobini E, Paoletti R, eds), pp 11-17. New York: Elsevier.

White CA, Sur M (1992) Membrane and synaptic properties of developing lateral geniculate nucleus neurons during retinogeniculate segregation. Proc Natl Acad Sci USA 89:9850-9854. 